Research Paper

\title{
4-1BBL has a Possible Role in Mediating Castration-Resistant Conversion of Prostate Cancer via Up-Regulation of Androgen Receptor
}

\author{
Hengcheng Zhu*, Min Wang ${ }^{* \bowtie}$, Yang Du, Xiuheng Liu, Xiaodong Weng, Chenglong Li \\ Department of Urology, Renmin Hospital of Wuhan University, Wuhan University, Jiefang Road 238, Wuhan 430060, Hubei, PR China \\ *Hengcheng Zhu and Min Wang contributed equally to this work and should be considered co-first authors \\ $\triangle$ Corresponding author: Min Wang, MD, PhD, Department of Urology, Renmin Hospital of Wuhan University, Wuhan, Hubei, PR China. E-mail: \\ drwangmin@163.com \\ (C) Ivyspring International Publisher. This is an open access article distributed under the terms of the Creative Commons Attribution (CC BY-NC) license \\ (https://creativecommons.org/licenses/by-nc/4.0/). See http://ivyspring.com/terms for full terms and conditions.
}

Received: 2018.09.02; Accepted: 2019.01.05; Published: 2019.06.02

\begin{abstract}
4-1BB ligand (4-1BBL) was a transmembrane glycoprotein belonging to the tumor necrosis factor family. It was expressed on activated $T$ lymphocytes and function as a co-stimulatory molecule via cross-linking with 4-1BB (a.k.a, CD137). In addition to its role in immune regulation, 4-1BBL transmitted signals into the cells on which it was expressed (reverse signaling). 4-1BBL represented a promising target for enhancing antitumor immune responses. Recent studies indicated that 4-1BBL also expressed in non-immune cells and possessed different functions in various types of cells. Here, we reported that 4-1BBL didn't express in normal prostate tissues and benign prostatic hyperplasia tissues, but it expressed in prostate cancer $(\mathrm{PCa})$ tissues at moderate level. Expression of 4-1BBL was up-regulated during the transition from PCa to castration resistant prostate cancer (CRPC). Increasing expression of 4-1BBL not only promoted expression of androgen receptor (AR), but also augmented proliferation and invasion ability of prostate cancer cells in androgen deprivation environment. These results were further verified by xenograft tumor experiments. Meanwhile, inhibiting AR signal pathway by chemical antagonist was able to significantly reduce 4-1BBL mediated proliferation and invasion of PCa cells. These novel findings indicated that 4-1BBL might mediate prostate cancer progression to castration-resistant prostate cancer via enhancing expression and function of $A R$.
\end{abstract}

Key words: 4-1BBL, prostate cancer, castration resistant prostate cancer, androgen receptor

\section{Introduction}

Prostate cancer (PCa) was considered as one of the most commonly diagnosed cancers in men of developed Western countries. Worldwide, it was the 2nd most commonly diagnosed and 6th leading cause of cancer death in men [1]. Androgen deprivation therapy (ADT) was still the standard systemic therapy for locally advanced or metastatic PCa. Growth of early stage of PCa required androgen and it could be successfully suppressed by ADT. However, almost all advanced PCa eventually progressed to a more aggressive form defined as castration resistant prostate cancer (CRPC), for which there was no available curative therapy [2].
The tumor necrosis factor receptor (TNFR)/TNF superfamily represented a key group of receptor/ ligands for controlling life and death in the immune system [3]. Among TNF superfamily members, 4-1BBL had emerged as an important mediator of survival signaling. It had been shown that $4-1 B B L$ expresses on mature B cells, macrophages, and dendritic cells. The interaction between 4-1BBL and its receptor 4-1BB provided a co-stimulatory signal to $\mathrm{T}$ cells, including activation of both $\mathrm{CD}^{+}$and $\mathrm{CD} 8^{+} \mathrm{T}$ cells, enhanced expansion $[4,5]$, increased long-term survival [6,7], and reduced apoptosis of activated $\mathrm{CD}^{+} \mathrm{T}$ cells[8]. In addition to acting as a ligand by cross-linking 4-1BB, 4-1BBL could receive signals and 
transmit them into the cells on which it was expressed, a process referred to as reverse signal transduction. Therefore, bidirectional signaling existed for the 4-1BB receptor/ligand system. The effects of reverse 4-1BBL signaling had received comparatively little attention in the past. However, during the last few years, a number of studies appeared to shed light on the biology of 4-1BBL signaling.

The prostate is an androgen-dependent organ in which organogenesis, morphology and normal functioning are regulated via the androgenic/androgen receptor (AR) signaling axis [9]. Recent evidence supports the notion that CRPC continued to rely on androgens via AR signaling [10]. Mechanisms that might contribute to restoring AR activity in CRPC include AR mutations or alternative splicing, increased intratumoral androgen synthesis, increased coactivator expression, and activation of several kinases that might directly or indirectly sensitize AR to low levels of androgens [11].

A number of studies had shown that 4-1BBL was also expressed on non-immunocytes. 4-1BBL was expressed on a wide range of tumor cells, including gastric, lung, laryngeal and liver cancer $[12,13]$. To our knowledge, little was known about the exact expression of 4-1BBL in human PCa. Here, this study investigated the distribution and expression of 4-1BBL in clinical human prostate tissues as well as PCa cell lines and xenograft tumor tissues. The aim was to enlighten the underlying involvement of 4-1BBL expression in PCa.

\section{Materials and Methods}

\section{Patients and Tissues}

The protocol was approved by the Ethics Committee of Renmin Hospital of Wuhan University (Jiefang Road 238, Wuhan 430060, Hubei PR, China), and the written informed consent was obtained from all patients. For immunohistochemical analysis, qRTPCR and western blotting analysis, samples from 22 cases of normal prostate, 30 cases of benign prostatic hyperplasia (BPH), 30 cases of androgen dependent prostate cancer (ADPC, primary and non-castration prostate cancer ) and 17 cases of CRPC tissue were collected from patients between September 2010 and June 2016. All prostate tissues of ADPC and CRPC were obtained from transurethral resection specimens.

\section{Cell Lines}

Human prostate cell lines RWPE-1, LNCaP, DU145, PC-3 and C4-2 were obtained from American Type Culture Collection (ATCC). The benign epithelial prostate cell line RWPE-1 was maintained in keratinocyte serum free media (Gibco). LNCaP,
DU145, PC-3 and C4-2 grown in RPMI 1640 (Life Technologies, CA) with 10\% FBS (Invitrogen). All cells were cultured in the incubator supplement with $5 \% \mathrm{CO}_{2}$ at $37^{\circ} \mathrm{C}$.

\section{Stable Transfection of PCa Cells with 4-1BBL Plasmid}

$3 \times 10^{6} \mathrm{LNCaP}$ cells were transfected with $2 \mu \mathrm{g}$ of pCDNA3.1-4-1BBL or empty vector and the C4-2 cells were transfected with $2 \mu \mathrm{g}$ of pGP-U6-4-1BBL-siRNA or empty vector by the mediation of $6 \mu \mathrm{L}$ Lipofectamine 2000 (Invitrogen, Carlsbad, CA, USA), according to the manufacturer's instructions. After 2 days of culture, the cells were replated into a $10 \mathrm{~cm}$ dish and cultured for another 2 days; complete RPMI-1640 medium containing $1000 \mu \mathrm{g} / \mathrm{mL}$ G418 (Sigma, St. Louis, MO) or Hygromycin (Sigma, St. Louis, MO) was added to the culture. After 20 days of selection, all nontransfected cells died, and discrete clones were visible in transfected cells. These clones were expanded in the presence of $200 \mu \mathrm{g} / \mathrm{ml} \mathrm{G} 418$ or Hygromycin; positive cells expressing 4-1BBL or not were named LNCap/4-1BBL and C4-2/si-4-1BBL. 4-1BBL mRNA expression was confirmed in positive cells after being selected.

\section{Xenograft tumor experiments}

Xenograft tumors were established using earlypassage cells and maintained in NOD/SCID mice. All animals were obtained from Beijing Hua Fukang Biological Technology Co. Ltd. and maintained in standard conditions according to the Institutional guidelines. The committee for experimental animals of Wuhan University approved all experimental procedures, and the procedures complied with the Guidelines for the Care and Use of Laboratory Animals. For s.c. tumor growth, $1 \times 10^{7} \mathrm{LNCaP}$ cells or LNCaP/4-1BBL cells were suspended in $75 \mu \mathrm{l}$ of RPMI- 1640 plus $75 \mu \mathrm{l}$ of Matrigel (Collaborative Biomedical Products, Bedford, MA) and injected via a 27-gauge needle into the s.c. space of the neck back region.

Mice were anesthetized with pentobarbital (45 $\mathrm{mg} / \mathrm{kg}$ ) and castrated (implemented with Bilateral orchidectomy) via scrotum incision approach. Animals were randomly divided into four groups (5 mice per group, $n=5)$ : 1) Control group, mice were injected with LNCaP cells without treated with castration; 2) Castration group, mice were injected with LNCaP cells, when subcutaneous implanted tumor grown up about 3 weeks, mice were treated with castration; 3) and 4-1BBL group, mice were injected with LNCaP/4-1BBL cells without treated with castration. According to our previous study [14], PCa had progressed to CRPC in vivo when 5 weeks 
after the mice being treated with castration. Then, all mice were killed, and tissues were collected for Western blot analysis and immune- histochemistry.

\section{Cell Counting Kit-8 assay}

Target cells $\left(2.5 \times 10^{5}\right.$ per well $)$ were cultured in 96 round bottom plates. After a 48-hour incubation at 37 ${ }^{0} \mathrm{C}$, the amount of released lactate dehydrogenase was determined by using Cell Counting Kit-8 (Dojindo, Japan) assay according to the manufacturer's instructions. All determinations were carried out in triplicate in 3 independent experiments.

\section{RNA extraction and qRT-PCR analyses}

Total RNA was extracted from prostate tissues and prostate cell lines using TRIzol reagent (Invitrogen, Grand Island, NY, USA) and cDNA was prepared using PrimeScript RT Reverse Transcriptase reagent Kit (Takara, Otsu, Japan). Quantitative RT-PCR (qRT-PCR) was performed on the Applied Biosystems 7300 real-time PCR system using SYBR Premix Ex TaqTM (Takara). The fold changes of 4-1BBL were analyzed by calculating the $2^{-\Delta \Delta \mathrm{Ct}}$ values. The same method was used for PCa cell lines and RWPE-1. Each sample was run in triplicates in 3 independent experiments.

\section{Western blot analysis}

Total proteins from prostate tissues and prostate cell lines were extracted. The rabbit polyclonal anti-41BBL antibody (Abcam, Cambridge, USA), rabbit polyclonal anti-AR antibody (Abcam, Cambridge, USA) and the HRP-conjugated anti-rabbit antibody (Abcam, Cambridge, USA) were used to probe Western blots. Mouse monoclonal anti- $\beta$-actin antibody (Cell signal technology, Boston, USA) and HRP conjugated goat anti-mouse IgG antibody (Cell signal technology, Boston, USA) were used to detect $\beta$-actin. Finally, the proteins were detected using an enhanced chemiluminescence system (ECL kit, Pierce Biotechnology, Beijing, China) and captured on lightsensitive X-ray film (Kodak, Shanghai, China). Optical densities were detected using ImageJ software.

\section{Immunohistochemistry}

The four-micrometer sections from formalinfixed and paraffin-embedded tissue blocks were dewaxed in xylene and rehydrated with ethanol. After blocked in goat serum, the sections were incubated with the anti-4-1BBL polyclonal antibody (Abcam, Cambridge, USA) or with the anti-KI67 polyclonal antibody (Abcam, Cambridge, USA) overnight at $4{ }^{\circ} \mathrm{C}$. Staining was then performed using Vectastain ABC Elite Kit (Vector Laboratories, Burlingame,CA), and sections were counterstained with hematoxylin.
By microscopic assessment, the 4-1BBL expression with immunostaining was positive in cell membrane and cytoplasm. Two pathologists who were blinded to clinical pathological outcome evaluated immunostaining reactions using a semiquantitative scoring system. The score about the percentage of positive cells $(0, \leq 5 \% ; 1,5-50 \% ; 2,>50 \%)$ and the score about the staining intensity (no staining; weak staining; moderate staining; and strong staining) were combined. If the total score was different between the two pathologists, an agreement was reached by rescoring the slides together with a third pathologist on a multi-viewer microscope.

\section{Invasion activity assay}

Transwell invasion assays were performed with $8.0 \mu \mathrm{m}$ pore according to the manufacturers' instructions (BD Bioscience, CA). The PCa cell invasion assays were performed with and coated Matrigel. The invaded PCa cells in the membrane were fixed and stained, and the cells were counted under a microscope. All determinations were carried out in triplicate in 3 independent experiments.

\section{Statistical analysis}

Statistical analyses were performed by SPSS 18.0 software. Student's t-test was performed for continuous variables and the chi-square test was used to analyze the differences of categorical variables. $\mathrm{P}<$ 0.05 indicated significant difference.

\section{Results}

\section{The expression of 4-1BBL is upregulated in clinical specimens of $\mathrm{PCa}$}

Immunohistochemistry was performed in all paraffin-embedded and archival normal prostate, BPH, ADPC and CRPC samples. The 4-1BBL expression was predominantly localized in cell membrane and cytoplasm, and the expression level was categorized as no staining, weak straining, moderate straining or strong straining (Figure 1). 4-1BBL was not detected in normal prostate and BPH samples, but it was detected in PCa samples. ADPC tissue showed moderate 4-1BBL expression, but ADPC tissue showed stronger 4-1BBL expression than in ADPC tissue.

To validate histology data, quantitative PCR was utilized to determine the mRNA level of $4-1 B B L$ in normal prostate, $\mathrm{BPH}$, and in several different human prostate cell lines including RWPE-1, LNCaP, DU145, PC-3 and C4-2. 4-1BBL mRNA was undetectable in normal prostate and BPH samples. While 4-1BBL mRNA expression was detected in ADPC samples, and its expression was significantly higher in CRPC tissues than in ADPC tissues $(\mathrm{P}<0.01)$ (Figure 2A). As 
shown in Figure 2B, the 4-1BBL mRNA was not detected in RWPE-1 cells, but it was detected in LNCaP, DU145, PC-3 and C4-2 cells. Furthermore, compared with DU145, PC-3 and C4-2 cells, expression of 4-1BBL mRNA was lower in LNCaP cells. And the 4-1BBL mRNA expression was significantly higher in C4-2 cell lines than in LNCaP, DU145 and PC-3 cell lines $(\mathrm{P}<0.01)$.

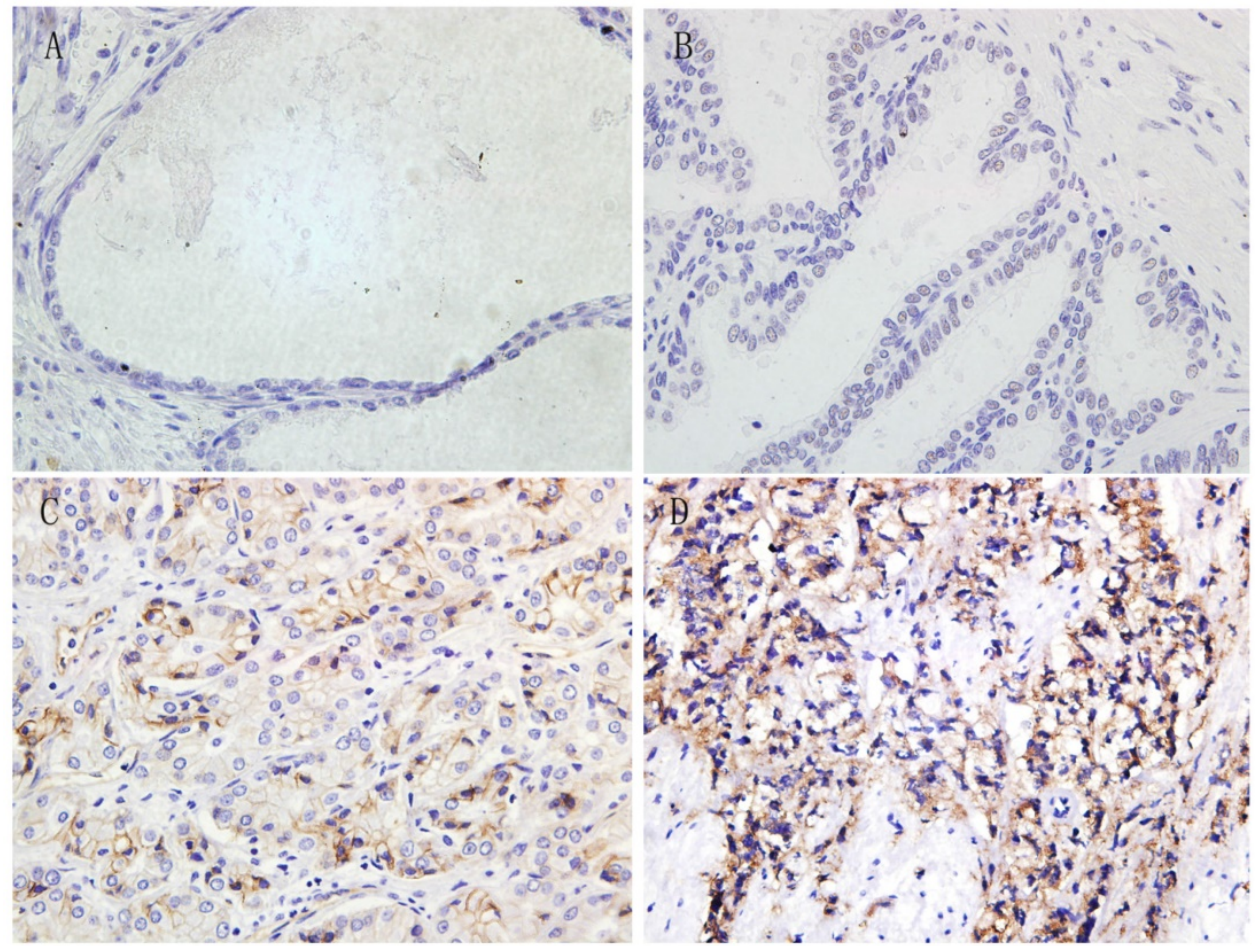

Figure 1. Immunohistochemical expression of 4-1BBL in Normal prostate, BPH, ADPC and CRPC tissues (magnification, 400). A, Normal prostate tissue showed no 4-1BBL expression. B, BPH tissue also showed no 4-1BBL expression. C, ADPC tissue showed moderate 4-1BBL expression. D, CRPC tissue showed strong 4-1BBL expression.
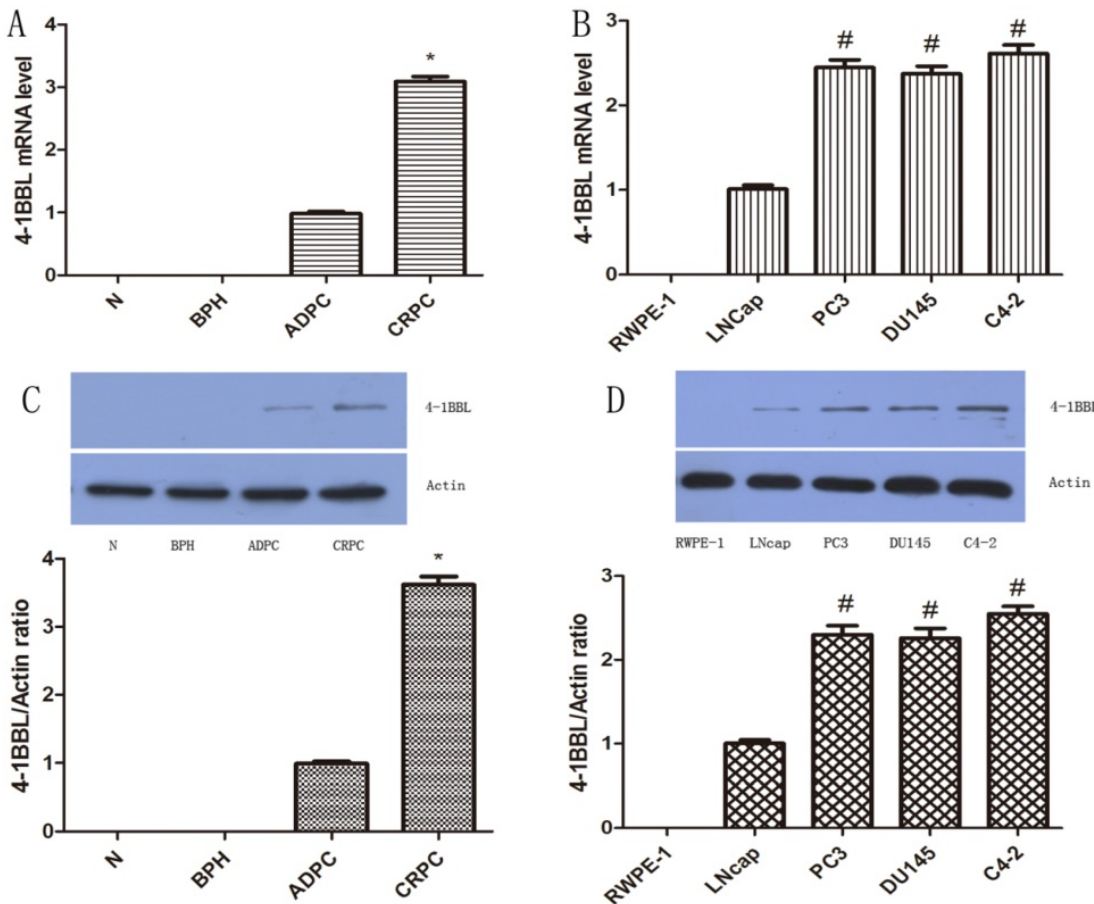

Figure 2. Expression of 4-1BBL in normal prostate, BPH, ADPC and CRPC tissues was confirmed at mRNA level by qRT-PCR and was confirmed at protein level by western blot. A, 4-1BBL mRNA expression was not detected in Normal prostate and BPH tissues, but the 4-1BBL mRNA expression was significantly higher in CRPC compared to ADPC tissue (*P<0.01 versus ADPC). B, 4-1BBL mRNA expression was not detected in RWPE-1, but it was higher in CRPC cell lines (PC3, DU145 and C4-2) than in ADPC cell lines (LNCaP). C, 4-1BBL expression was not detected in Normal prostate and BPH tissues, but the 4-1BBL expression was significantly higher in CRPC compared to ADPC tissue $(* P<0.01$ versus ADPC). D, 4-1BBL expression was not detected in RWPE-1, but it was higher in CRPC cell lines (PC3, DU145 and C4-2) than in ADPC cell lines (LNCaP). In addition, 4-1BBL expression was highest in $\mathrm{C} 4-2$ cell line (\#P<0.01 versus LNCaP). (N: Normal prostate; BPH: Benign prostate hyperplasia; ADPC: Androgen dependent prostate cancer; CRPC: Castration resistant prostate cancer) 
We further checked the protein level of 4-1BBL in normal prostate, BPH, ADPC and CRPC samples and in Human prostate cell lines RWPE-1, LNCaP, DU145, PC-3 and C4-2. The protein expression of 4-1BBL was assessed by western blot (Figure 2C and Figure 2D). The results were consistent with data from IHC and qRT-PCR assay $(\mathrm{P}<0.01)$.

\section{Overexpression of 4-1BBL increased proliferation and invasion of PCa cells in androgen deprivation environment}

To determine the function of 4-1BBL on proliferation ability of $\mathrm{LNCaP}$ cells. LNCaP cells were transfected with pCDNA3.1 and pCDNA3.1/4-1BBL, and the G418-resistant cells (LNCaP/pCDNA3.1, $\mathrm{LNCaP} / 4-1 \mathrm{BBL})$ were selected. The cells were cultured in androgen deprivation environment (phenol red free RPMI1640 medium with activated Charcoal/ Dextran treated FBS). As shown in Figure 3A, $\mathrm{LNCaP} / 4-1 \mathrm{BBL}$ cells displayed higher proliferation than LNCaP cells and LNCaP/pCDNA3.1 cells in androgen deprivation environment assessed by CCK8 assay. Meanwhile, the invasion ability of $\mathrm{LNCaP} /$ 4-1BBL cells was higher than LNCaP cells and LNCaP/pCDNA3.1 cells in androgen deprivation environment assessed by Transwell invasion assays (Figure 3B).

\section{Overexpression of 4-1BBL upregulated the expression of $A R$ in vitro}

LNCaP cells were transfected with pCDNA3.1/ 4-1BBL, and the G418-resistant cells (LNCaP/4-1BBL) were selected. Meanwhile, C4-2 cells were transfected with pGP-U6-4-1BBL- siRNA, and the Hygromycinresistant cells (pGP-U6-4-1BBL-siRNA) were selected. Then, mRNA expression of 4-1BBL was determined by qRT-PCR to infirm the transfection efficiency and protein expression of AR was assessed by western blot. The results showed that mRNA expression of 4-1BBL was significantly increased in LNCap/4-1BBL cells compared with in LNCap cells $(\mathrm{P}<0.05)$, while mRNA expression of 4-1BBL was significantly decreased in C4-2/si-4-1BBL cells compared with in C4-2 cells $(\mathrm{P}<0.05)$ (Figure $4 \mathrm{~A})$. On the other hand, protein expression of AR was increased in LNCap/ 4 -1BBL cells compared with in LNCap cells $(\mathrm{P}<0.05)$, while protein level of AR was decreased in C4-2/si-4-1BBL cells compared with in C4-2 cells $(\mathrm{P}<0.05)$ (Figure 4B). The change of $\mathrm{AR}$ protein expression was consistent with the change of 4-1BBL expression. These data suggest that 4-1BBL may play a role in PCa via upregulating AR expression.
B

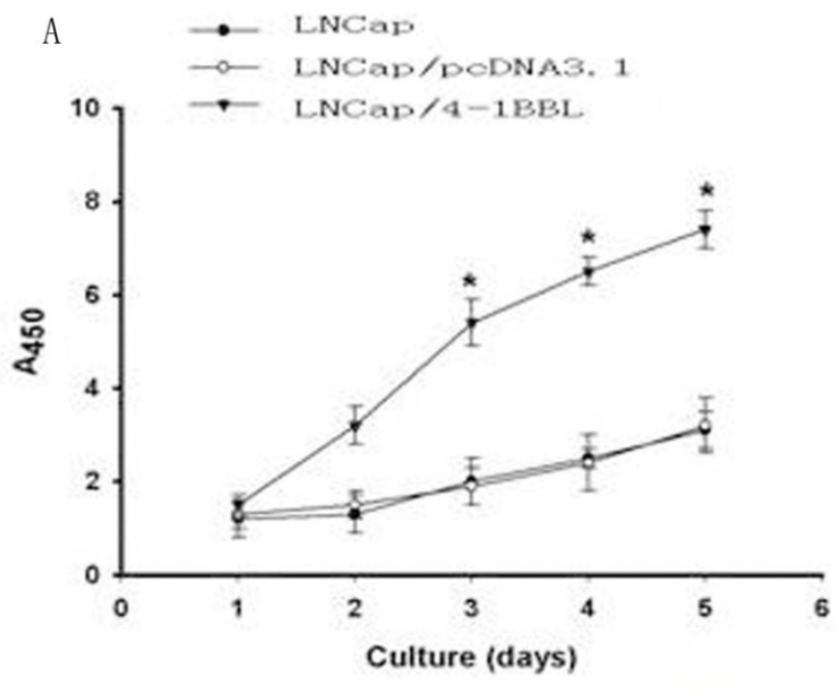

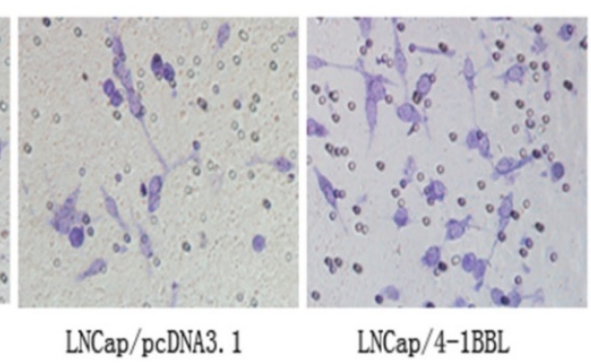

LNCap/pcDNA3. 1

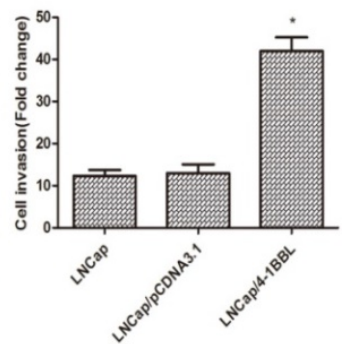

Figure 3. Function of 4-1BBL on proliferation ability and invasion ability of LNCaP cells in androgen deprivation environment. A, LNCaP cells transfected with PCDNA3.1/4-1BBL still demonstrates strong proliferation ability in phenol red free RPMI1640 medium with activated Charcoal/Dextran treated FBS. B, LNCaP cells transfected with PCDNA3.1/4-1BBL demonstrates stronger invasion ability than LNCaP or LNCaP/ PCDNA3.1 cells in phenol red free RPMI1640 medium with activated Charcoal/Dextran treated $\mathrm{FBS})$. (*P<0.05 versus $\mathrm{LNCaP}$ or $\mathrm{LNCaP} / \mathrm{PCDNA3.1)}$. 

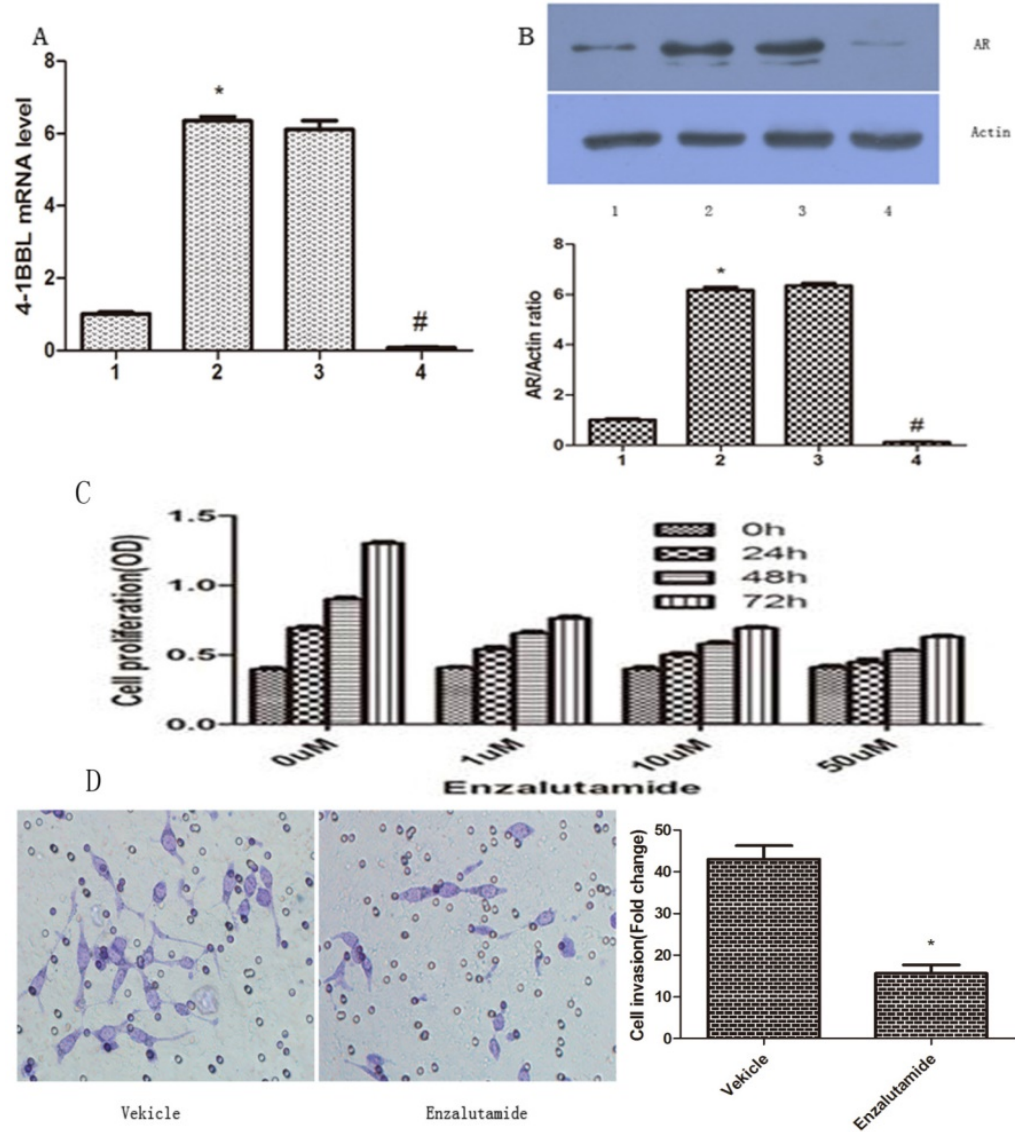

Figure 4. Effect of 4-1BBL on AR expression in PCa cells was confirmed by $q R T-P C R$ and by western blot. A, 4-1BBL mRNA expression was significantly higher in LNCap/4-1BBL cells compared to LNCap cells, while 4-1BBL mRNA expression was significantly lower in C4-2/si-4-1BBL cells compared to C4-2 cells (1: LNCap cell; 2: LNCap/4-1 BBL cell; 3: C4-2 cell; 4: C4-2/si-4-1BBL cell). B, AR expression was significantly higher in LNCap/4-1BBL cells compared to LNCap cells, while AR expression was significantly lower in C4-2/si-4-1BBL cells compared to C4-2 cells. ( $* \mathrm{P}<0.05$ versus $\mathrm{LNCap}$; $\# \mathrm{P}<0.05$ versus $\mathrm{C} 4-2$ ). C, Proliferation ability of LNCap/4-1BBL cells influenced by Enzalutamide was assessed by CCK8 assay. Enzalutamide (an inhibitor of AR signaling) decreased proliferation ability of LNCaP cells induced by 4-1BBL. D, Invasion ability of LNCap/4-1BBL cells influenced by Enzalutamide was assessed by Transwell invasion assay. Enzalutamide reduced invasion ability of LNCaP cells induced by 4-1BBL. (Enzalutamide group Vs Vekicle group, $\mathrm{P}<0.05)$

\section{Enzalutamide inhibits proliferation and invasion of 4-1 BBL overexpressing PCa cells}

To further confirm if 4-1BBL functions through AR, we applied Enzalutamide (a synthetic nonsteroidal anti-androgen) to the medium of $\mathrm{PCa}$ cultures. The proliferation ability of LNCaP/4-1BBL were assessed after being treated with $0 \mu \mathrm{M}, 1 \mu \mathrm{M}, 10$ $\mu \mathrm{M}$ or $50 \mu \mathrm{M}$ Enzalutamide (Sigma, USA) for $0 \mathrm{~h}, 24 \mathrm{~h}$, $48 \mathrm{~h}$ or $72 \mathrm{~h}$, respectively. The results revealed that Enzalutamide significantly blocked the proliferation (Figure 4C) and invasion (Figure 4D) ability of LNCaP cells overexpressing 4-1BBL. Thus, our data confirmed that 4-1BBL contributes to progression of PCa via enhancing AR expression.

\section{4-1BBL expression was up-regulated when $P C a$ progressed to CRPC in vivo, and overexpression of 4-1BBL promoted proliferation of $P C a$ cells and increased the expression of $\mathbf{A R}$}

As shown in Fig 5A, 4-1BBL expression was significantly up-regulated in the Castration group compared with in the Control group. In the 4-1BBL group, 4-1BBL expression was significantly higher than in the Control group $(\mathrm{P}<0.05)$, and $4-1 \mathrm{BBL}$ expression was also significantly higher than in the Castration group $(\mathrm{P}<0.05)$. The level of AR expression in the Castration group increased significantly compared with that in the Control group $(\mathrm{P}<0.05)$. Furthermore, the expression of $\mathrm{AR}$ in the 4-1BBL group was significantly higher than that in the Castration group $(\mathrm{P}<0.05)$.

$\mathrm{KI}-67$ is a nuclear non-histone protein, and it is a well-known proliferation marker that is widely used in the diagnosis of several pathologic lesions, especially human tumors and malignancies[15]. 35 days after castration, the expression of KI67 was consistent with the expression of $4-1 \mathrm{BBL}$ in the Control group, in the Castration group and in the 4-1BBL group Fig 5B. The expression of KI67 was significantly higher in the Castration group tissues than in the Control group tissues $(\mathrm{P}<0.05)$, meanwhile, the expression of KI67 was a little higher in the 4-1BBL group than in the Castration group $(\mathrm{P}<0.05)$. 

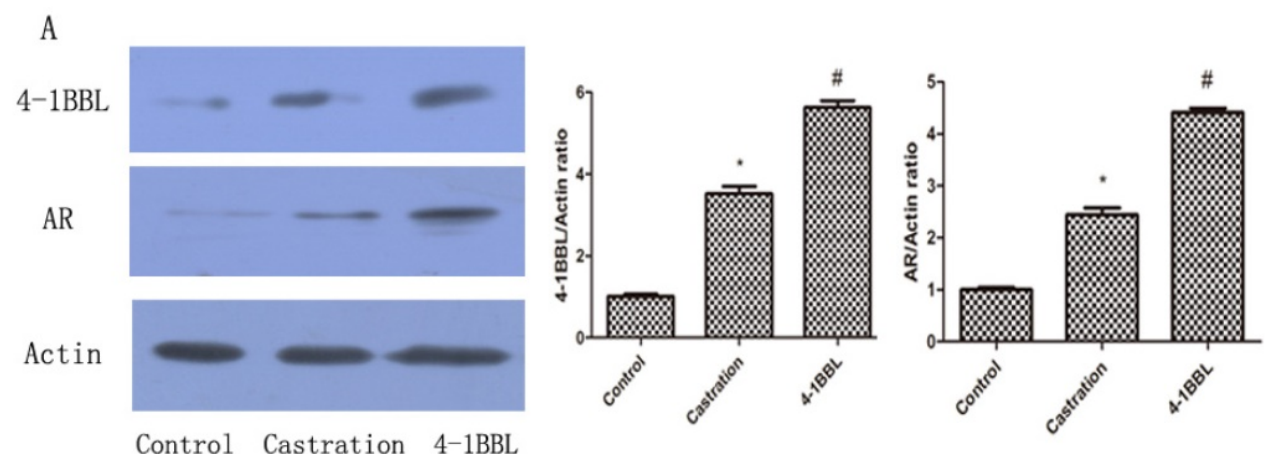

B

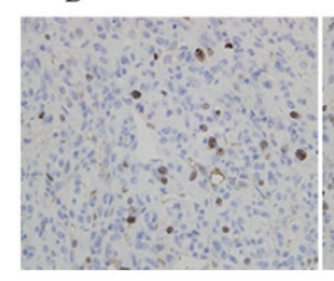

Control

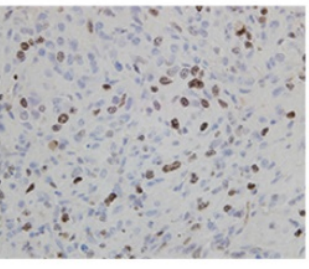

Castration

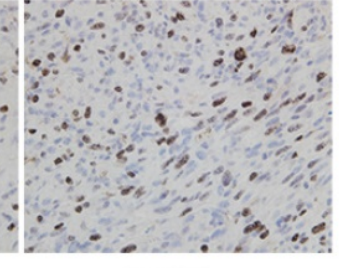

4-1BBL

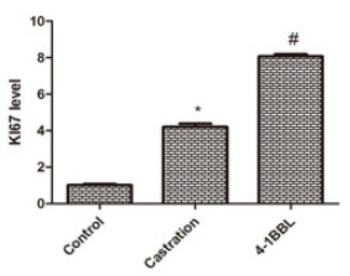

Figure 5. A, Expressions of 4-1BBL and AR in Control group, Castration group and 4-1BBL group were confirmed at protein level by western blot. Both 4-1BBL and AR expressions were significantly up-regulated in the Castration group than in the Control group $(\mathrm{P}<0.05)$. In the 4-1BBL group, both $4-1 \mathrm{BBL}$ and $\mathrm{AR}$ expressions were significantly higher compared to the Control group $(\mathrm{P}<0.05)$, and both 4-1BBL and AR expressions were significantly higher than in the Castration group $(\mathrm{P}<0.05)$. $B$, Immunohistochemical expression of KI67 in Control group, Castration group and 4-1BBL group tissues (magnification, 400). The expression of KI67 was significantly higher in the Castration group tissues than in the Control group tissues $(P<0.05)$, furthermore, the expression of K167 was significantly higher in the 4-1BBL group than in the Castration group $(P<0.05)$.

\section{Discussion}

4-1BBL had been shown to be mainly expressed on activated T cells, and its best characterized activity was $\mathrm{T}$ cell co-stimulation. Recently, 4-1BBL was also found to be expressed on carcinoma cells, and it might significantly influence the outcome of a T cell-tumor cell interaction. Thus far, no study had reported the precise role of $4-1 \mathrm{BBL}$ in PCa. This study was interested to see whether there was an aberrant expression of 4-1BBL in $\mathrm{PCa}$, and the function of 4-1BBL played in PCa development and progression.

$4-1 \mathrm{BB}$ was a member of the TNFR family and could be expressed by a range of leucocytes, including activated $\mathrm{T}$ cells, natural killer (NK) cells and inflamed vascular endothelial cells [15]. 4-1BBL belonged to the TNF family, and it was expressed widely and constitutively, including by antigen presenting cells (APC) as a transmembrane protein on the cell surface. $4-1 \mathrm{BBL}$ was also expressed by many nonimmune cells as a transmembrane protein on the cell surface. In one hand, 4-1BBL acted as a ligand by cross-linking 4-1BB to co-stimulate $T$ cell activation. In the other hand, 4-1BBL could receive and transmitted signals into the cells on which it was expressed, this process referred to as reverse signal [16].

In the present study, a cohort of $\mathrm{PCa}, \mathrm{BPH}$ and normal prostate samples were examined by histology. 4-1BBL was not detected in normal prostate and $\mathrm{BPH}$ samples, but it was detected in PCa samples. Meanwhile, the 4-1BBL expression was higher in
CRPC than in primary PCa. To further validate these findings, qRT-PCR and western blot were employed to examine the expression of 4-1BBL at mRNA and protein levels in $\mathrm{PCa}, \mathrm{BPH}$ and normal prostate tissues as well as five prostate cell lines. The results supported the notion that expression of 4-1BBL was clearly elevated in PCa tissues, and were positively related with PCa progression to CRPC. Analysis of PCa cell lines further showed that the mRNA and protein levels of 4-1BBL were significantly upregulated in androgen-independent prostate cancer cell lines than in androgen-dependent prostate cancer cell lines. Furthermore, 4-1BBL expression was significantly up-regulated when PCa had progressed to CRPC about 35 days after castration in vivo. This was consistent with the previous report that 4-1BBL was more common in malignant tumors, especially in moderate or low-differentiated tumors [17].

To date, ADT remained as the standard therapy for men with de novo or recurrent metastatic disease [18]. Typically, ADT was able to reduce the level of prostate specific antigen (PSA) in $80-90 \%$ of patients during initial treatment. However, almost all patients virtually progressed to CRPC at a median of 18-24 months after diagnosis [19]. There was no curative therapy available for CRPC. In a large portion of castration-resistant tumors, an enhanced expression of the AR was detected, which could result in a reactivation of $\mathrm{AR}$ transcriptional activity despite low androgen levels. In $20-30 \%$ of CRPC tumors the enhanced AR expression was based on AR gene 
amplifications [20, 21]. This study revealed that overexpression of 4-1BBL significantly augmented proliferation and invasion of androgen dependent PCa cells in androgen deprivation environment. Meanwhile, both expression of AR in prostate cancer cell lines and in PCa tumor bearing SCID mice were positively associated with overexpression of 4-1BBL. Furthermore, 4-1BBL mediated increase of proliferation and invasion abilities of $\mathrm{LNCaP}$ could be blocked by Enzalutamide, a specific AR signaling inhibition. Therefore, this study speculated that 4-1BBL might play a critical role in PCa progression to CRPC via enhancing AR function.

The 4-1BB/4-1BBL pair emerged as a target for PCa treatment due to its ability to enhance the generation of effective tumor-specific immune responses, which appeared to be primarily involved in the induction of apoptosis of tumor cells under certain conditions. 4-1BBL on some tumor cells was capable of triggering tumor cells to produce IL-8, which is critical for the development and progression of numerous malignancies [22]. Results of this study were consistent with these publications.

To our knowledge, this was the first time that the common up-regulation of 4-1BBL was identified in human PCa and normal prostate tissues. Furthermore, our data revealed a positive correlation between increased expression of 4-1BBL and PCa progression to CRPC. In addition, this study discovered that 4-1BBL was quite closely related to PCa cells proliferation and contributed to PCa progression to CRPC might via up-regulating expression and activity of AR. Thus, 4-1BBL may be a therapeutic target for treatment of CRPC.

\section{Acknowledgements}

We are grateful to Staffs in Key laboratory of Hubei Province for Digestive System Disease for their kind assistance. This study was supported by the grants from the National Natural Science Foundation of China (No.2013RMFH012), the Province Natural Science Foundation of Hubei (No.2012FFA096), the Province Natural Science Foundation of Hubei (No.20 16CFB114), the Science Foundation of Wuhan (NO.20 15060101010049) and supported by the Fundamental Research Funds for the Central Universities (NO.2042 017kf0089).

\section{Competing Interests}

The authors have declared that no competing interest exists.

\section{References}

[1] Jemal A, Bray F, Center MM, et al. Global cancer statistics. CA Cancer J Clin. 2011; 61: 69-90.
[2] Larsson R, Mongan NP, Johansson M, et al. Clinical trial update and novel therapeutic approaches for metastatic prostate cancer. Curr Med Chem. 2011; 18: $4440-4453$.

[3] Locksley RM, Killeen N, Lenardo MJ. The TNF and TNF receptor superfamilies: integrating mammalian biology. Cell. 2001; 104: 487-501.

[4] Laderach D, Movassagh M, Johnson A, et al. 4-1BB co-stimulation enhances human CD8(+) T cell priming by augmenting the proliferation and survival of effector CD8(+) T cells. Int Immunol. 2002; 14: 1155-1167.

[5] Lu ZY, Condomines M, Tarte K, et al. B7-1 and 4-1BB ligand expression on a myeloma cell line makes it possible to expand autologous tumor-specific cytotoxic T cells in vitro. Exp Hematol. 2007; 35: 443-453.

[6] Lee HW, Park SJ, Choi BK, et al. 4-1BB promotes the survival of CD8+ T lymphocytes by increasing expression of Bcl-xL and Bfl-1. J Immunol. 2002; 169: 4882-4888.

[7] Croft M. Co-stimulatory members of the TNFR family: keys to effective T-cell immunity? Nat Rev Immunol. 2003; 3: 609-620.

[8] Kudo-Saito C, Hodge JW, Kwak H, et al. 4-1BB ligand enhances tumor-specific immunity of poxvirus vaccines. Vaccine. 2006; 24: 4975-4986.

[9] Schaeffer EM, Marchionni L, Huang Z, et al. Androgen- induced programs for prostate epithelial growth and invasion arise in embryogenesis and are reactivated in cancer. Oncogene. 2008; 27: 7180-7191.

[10] Mellado B, Codony J, Ribal MJ, et al. Molecular biology of androgenindependent prostate cancer: the role of the androgen receptor pathway. Clin Transl Oncol. 2009; 11: 5-10.

[11] Yuan X, Balk SP. Mechanisms mediating androgen receptor reactivation after castration. Urol Oncol. 2009; 27: 36-41.

[12] Salih HR, Kosowski SG, Haluska VF, et al. Constitutive expression of functional 4-1BB (CD137) ligand on carcinoma cells. J Immunol. 2000; 165: 2903-2910

[13] Cheng K, Wong SC, Linn YC, et al. CD137 ligand signaling induces differentiation of primary acute myeloid leukaemia cells. Br J Haematol. 2014; 16: 134-144.

[14] Wang M, Liu X, Guo J, et al. Inhibition of LSD1 by Pargyline inhibited process of EMT and delayed progression of prostate cancer in vivo. Biochem Biophys Res Commun. 2015; 13: 310-315.

[15] Mateoiu C, Pirici A, Bogdan F. Immunohistochemical nuclear staining for p53, PCNA, Ki-67 and bcl-2 in different histologic variants of basal cell carcinoma. Rom J Morphol Embryol. 2011;52:315-319.

[16] Thum E, Shao Z, Schwarz H. CD137, implications in immunity and potential for therapy. Front Biosci. 2009; 14: 4173-4188.

[17] Eissner G, Kolch W, Scheurich P. Ligands working as receptors: reverse signaling by members of the TNF superfamily enhance the plasticity of the immune system. Cytokine Growth Factor. 2004; 15: 353-366.

[18] Dimberg J, Hugander A, Wågsäter D. Expression of CD137 and CD137 ligand in colorectal cancer patients. Oncol Rep. 2006; 15: 1197-1200.

[19] Assikis VJ, Simons JW. Novel therapeutic strategies for androgen-independent prostate cancer: an update. Semin Oncol. 2004; 31: 26-32.

[20] Harris WP, Mostaghel EA, Nelson PS, et al. Montgomery, Androgen deprivation therapy: progress in understanding mechanisms of resistance and optimizing androgen depletion. Nat Clin Pract Urol. 2009; 6: 76-85.

[21] Koivisto P, Kononen J, Palmberg C, et al. Androgen receptor gene amplification: a possible molecular mechanism for androgen deprivation therapy failure in prostate cancer. Cancer Res. 1997; 57: 314-319.

[22] Bubendorf L, Kononen J, Koivisto P, et al. Survey of gene amplifications during prostate cancer progression by high throughout fluorescence in situ hybridization on tissue microarrays. Cancer Res. 1999; 59: 803-806.

[23] Brat DJ, Bellail AC, Van Meir EG. The role of interleukin-8 and its receptors in gliomagenesis and tumoral angiogenesis. Neuro Oncol. 2005; 7: 122-133. 